\title{
KONGRESSKALENDER
}

\section{August 2018}

28.-31.08.2018, Vilnius, Litauen

15th Conference International Association for the Treatment of Sexual Offenders (IATSO)

Information: www.iatso.org

29.08-01.09.2018, Sarajevo, Bosnien

European Association of Criminology

18th Annual ESC Conference

Information: http://www.esc-eurocrim.org/

\section{September 2018}

24.09.2018, Essen

Symposium: Aktuelle Forschungsbereiche der Forensischen Psychiatrie

Institut für Forensische Psychiatrie der

Universität Duisburg Essen - LVR Klinikum

Essen

Prof. Dr. N. Leygraf

Information: www.forensik-essen.de;

claudia.enders@1vr.de

\section{Oktober 2018}

\section{1.-13.10.2018, München}

33. Herbsttagung für Forensische Psychiatrie (AGFP)

Abteilung für Forensische Psychiatrie der Universität München

Prof. Dr. K. Schiltz

Information: www.forensik-muenchen.de

17.-20.10.2018, Vancouver, BC, Canada Association for the Treatment of Sexual Abusers (ATSA)

Hyatt Regency Vancouver

Information: http://www.atsa.com/future-

atsa-conferences

\section{3.-25.10.2018, Bergen, Norwegen}

5th Bergen International Conference on Forensic Psychiatry

Neurocognitive Disorders Across the Lifespan

Information: www.bergenconference.no
25.-26.10.2018, Wiesbaden, KrimZ

Herbsttagung: Missbrauch und Zwang im institutionellen Kontext

PD Dr. M. Rettenberger, Prof. Dr. A.

Dessecker

Kriminologische Zentralstelle (KrimZ)

Information: www.krimz.de/tagungen

25.-28.10.2018, Austin, Texas (USA)

American Academy of Psychiatry and the Law

49th Annual Meeting (with APA)

JW Marriott Austin

Information: www.AAPL.org

\section{November 2018}

14.-17.11.2018 Atlanta, GA (USA)

American Society of Criminology Annual Meeting

Institutions, Cultures, and Crime.

Atlanta Marriott Marquis

Information: http://www.asc41.com/

annualmeeting.htm

\section{März 2019}

06.-08.03.2019, Lippstadt-Eickelborn 34. Eickelborner Fachtagung zu Fragen der Forensischen Psychiatrie

Kontaktadresse: Michaela.Riepe@ wkp-lwl.org

Information: www.lwl-forensik-lippstadt.de

20.-22.03.2019, Potsdam-Griebnitzsee

20. Basiskurs Forensische Psychiatrie:

Schwerpunkt Kriminalprognose

Prof. Dr. H.-L. Kröber, Dr. F. Wendt

Zentrum für Forensisch-Psychiatrische

Begutachtung

Information:www.zfpb.de; kroeber@zfpb.de

\section{Mai 2019}

31.05.2019, Dresden

24. Dresdner Frühjahrstagung für

Forensische Psychiatrie

Dr. Lange, Prof. Dr. Bauer, Dr. Lammel, Dr. Sutarski

Information: www.forensik-dresden.de

\section{Juni 2019}

05.-07.06.2019, Zürich, Schweiz

11. Internationales Symposium

Forensische Psychiatrie

World Trade Center

Prof. Endrass, Dr. Rossegger, Prof. Urbaniok

Information: www.forensiktagung.ch

21.06.2019, Berlin-Mitte

23. Berliner Junitagung für Forensische

Psychiatrie und Psychologie

Forensische Psychiatrie Psychologie Berlin e.V. (FPPB) \&

Institut für Forensische Psychiatrie der Charité

Langenbeck-Virchow-Haus

Information: www.forensik-berlin.de

(Veranstaltungen)

\section{September 2019}

28.-30.09.2019, Wien

16. Wissenschaftliche Fachtagung der Kriminologischen Gesellschaft (KrimG)

Prof. Dr. Chr. Grafl

Information: www.krimg.de

Hinweise für den Kongresskalender bitte an: mail@hlkroeber.de 